\title{
Severe coronary artery stenosis is associated with poor neurological outcome in cardiac arrest survivors: a multi-center retrospective cohort study
}

Chih-Wei Sung ${ }^{1}$; Joyce Tay ${ }^{2}$; Meng-Che Wu ${ }^{1}$; Jia-How Chang ${ }^{1}$; Chien-Hua Huang ${ }^{2}$; Wen-Jone Chen ${ }^{2}$; Wei-Tien Chang ${ }^{2}$; Min-Shan Tsai ${ }^{1,2}$

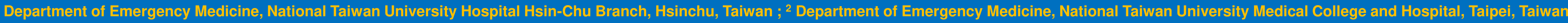

PURPOSE

The association of coronary artery stenosis and poor neurological outcome is unknown.

The aim of the study is to find the association between the coronary artery stenosis and the neurological outcome in patients with cardiac arrest.

\section{METHODS}

A total of 273 non-traumatic adult cardiac arrest patients who achieved return of spontaneous circulation (ROSC) and underwent emergent coronary angiography (CAG) during the period from 2011 to 2017 were retrospectively recruited from three hospitals. The severity of coronary artery lesion during CAG, percutaneous coronary intervention, baseline characteristics and events of cardiopulmonary resuscitation were collected and compared between patients with and without favorable neurological outcome, which was classified by cerebral performance scale (CPC).

\section{RESULTS}

A total of 273 patients was divided into four groups based on their vessel number of coronary artery stenosis. One hundred and ten patients out of 135 patients (81\%) with poor neurological outcome $(\mathrm{CPC}=3 \sim 5)$. had at least 1-vessel coronary artery severe stenosis ( $\geq 70 \%$ stenosis). The baseline characteristic of these groups was shown in Table 1 . When compared with those with patent coronary artery, the patients with coronary artery stenosis had a significant higher risk for poor neurological outcome which positively correlated with the number of vessels (1-vessel: adjusted $\mathrm{HR} 1.29,95 \% \mathrm{Cl} 0.62-$ 2.66, $p=0.081$; 2-vessel: adjusted HR 2.33, 95\% Cl 1.09-4.94, $p=$ 0.028; 3-vessel: adjusted HR 3.99, 95\% Cl 1.84-8.68, $p<0.001$ ).

\section{CONCLUSIONS}

The severity of coronary artery stenosis is associated with neurological outcome in cardiac survivors.

CONFLICT OF INTEREST

The authors declared that they have no conflict of interest like financial interests, activities, and affiliations.
FIG 1. PATIENT SELECTION

3132 non-traumatic adult cardiac arrest
patients with CPR attempt at ER (IHCA \&
OHCA)
OHCA)

\section{4 patients with sustained ROSC}

\section{0 patients sent to cardiac} catheterization room

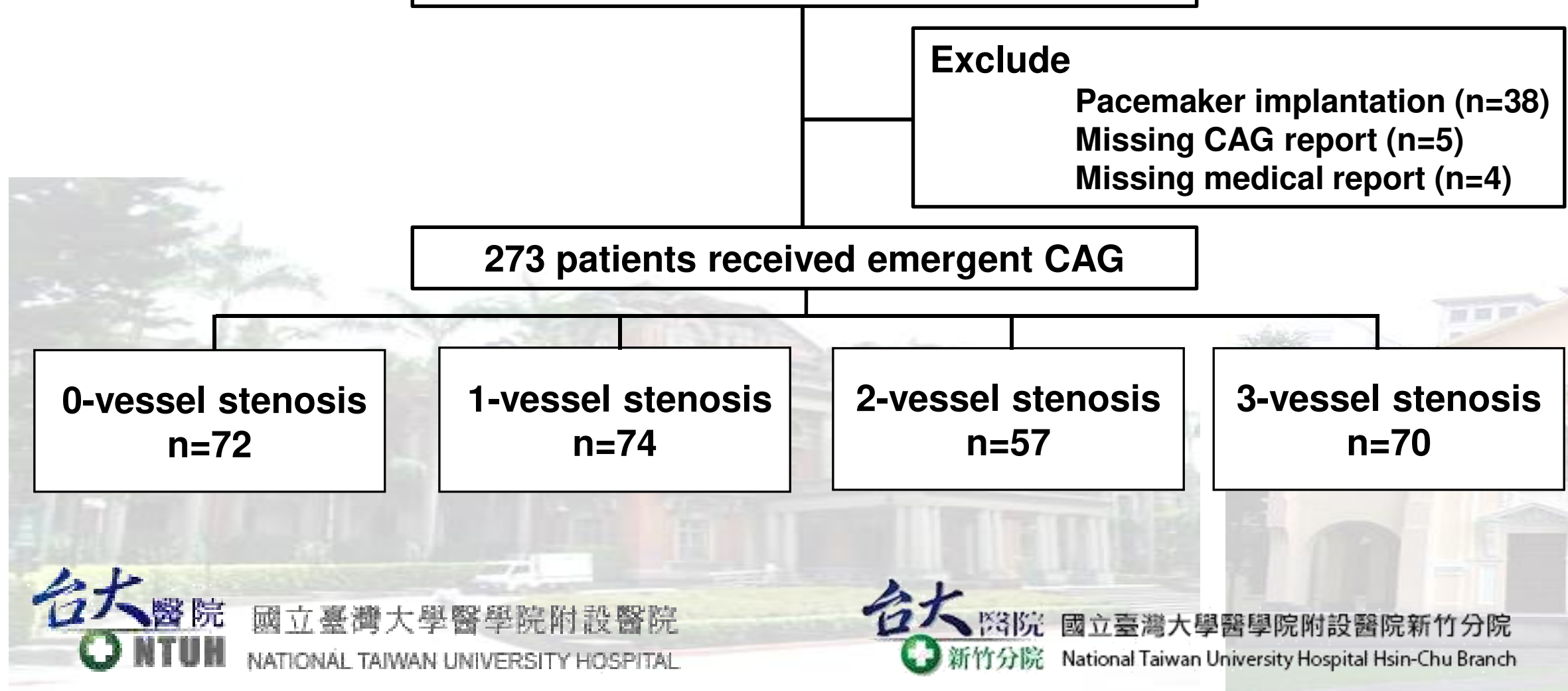

TABLE 1. BASELINE CHARACTERISTICS

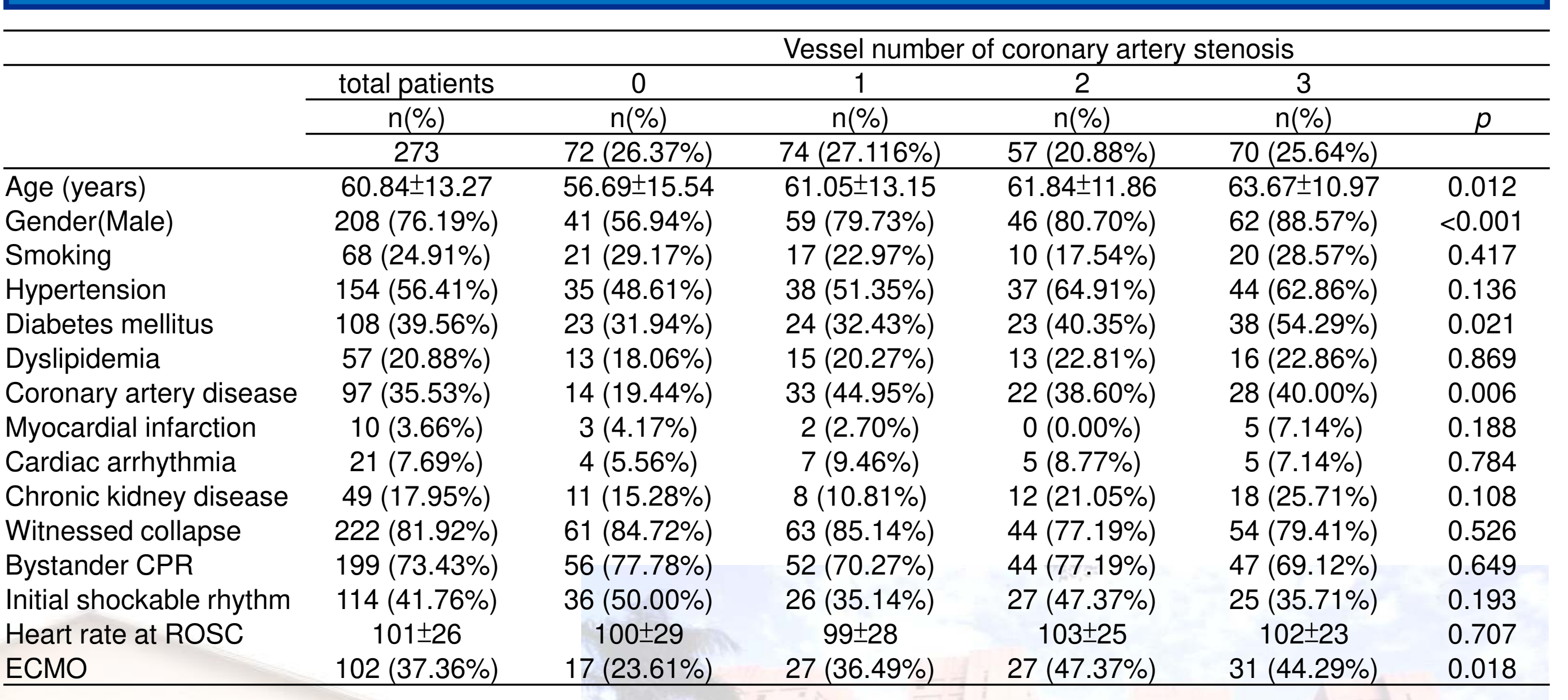

\section{TABLE 2. NEUROLOGICAL OUTCOME}

Poor neurological outcome $\quad$ Unadjusted $\mathrm{HR}(95 \% \mathrm{Cl}) \quad \mathrm{p} \longrightarrow$ Adjusted $\mathrm{HR}(95 \% \mathrm{Cl}) \quad p$ Vessel number of coronary artery stenosis $\begin{array}{cc}\text { Vessel number of coronary artery steno } \\ 0 & 25(18.52 \%)\end{array}$ 